\title{
An unusual case of gonococcal arthritis
}

\author{
K M RENNEY \\ From the Department of Family Practice, San Bernardino County Medical Center, San Bernardino, \\ California, USA
}

SUMMARY In cases of gonococcal arthritis the knees, wrists, ankles, hands, and feet are the joints most commonly affected. This case is unusual because of the occurrence of gonococcal arthritis in the sacroiliac joint.

\section{Introduction}

Arthritis is a common complication of gonococcal infection. The incidence is about $1 \%$ in patients who develop gonorrhoea. ${ }^{1}$ The joints most commonly affected are the knees, wrists, ankles, and the metacarpophalangeal and metatarsophalangeal joints. ${ }^{2}$ In the present case gonococcal arthritis of the right sacroiliac joint was found, which is an unusual occurrence.

\section{Case report}

A 21-year-old black woman was admitted on 26 September 1976 with a seven-day history of migratory joint pains finally resulting in severe pain in the right hip. The patient stated that the joint pain had begun in the right thumb and had progressed to her left ankle, and right hip, knee, and ankle. Each joint had become swollen, reddened, and very tender for limited periods of time. She denied any recent history of upper respiratory illness, kidney infection, or exposure to gonorrhoea or syphilis.

On clinical examination the patient was in moderate distress. Her temperature was $37 \cdot 4^{\circ} \mathrm{C}$, pulse rate 96 beats $/ \mathrm{min}$, and blood pressure $140 / 88 \mathrm{~mm} \mathrm{Hg}$. On examination of the joints and extremities there was no increased warmth, redness, or effusion over any previously affected joints. The range of movement of the right hip was limited because of pain and there was point tenderness over the right sacroiliac region. The patient was unable to bear weight on the right leg owing to the severe pain in the right hip with radiation to the sacral region.

White blood count was $7.7 \times 10^{3} / \mathrm{mm}^{3}$ $\left(7 \cdot 7 \times 10^{9} / \mathrm{l}\right)$ with $71 \%$ neutrophils, packed cell

Address for reprints: Dr K M Renney, 7651 Highland Woods CT/2b, Lorton, Virginia 22079, USA

Received for publication 17 January 1979 volume $36 \cdot 5 \%$, and erythrocyte sedimentation rate $37 \mathrm{~mm} / \mathrm{h}$. The ASO titre was reported as 50 Todd units. Additional investigations giving negative results were antinuclear antibodies (ANA), lupus erythematosus cells, rheumatoid factor, Monotest, Venereal Disease Research Laboratory test, DriDot pregnancy test, and sickle-cell screen. Urine analysis showed 5-7 polymorphonuclear leucocytes per high power field and acetone. Electrolyte levels were normal; uric acid was $0.09 \mathrm{mmol} / 1$ and salicylate level was $2 \cdot 64 \mathrm{nmol} / \mathrm{l}$.

Radiographs of the chest, lumbosacral spine, sacroiliac joint, pelvis, and right hip showed no abnormalities. An electrocardiogram was normal.

After specimens for culture for gonococci from the cervix, vagina, and rectum, together with specimens of blood and urine, had been obtained, the patient was rested in bed and treated with acetylsalicylic acid $600 \mathrm{mg}$ every four hours.

An orthopaedic surgeon confirmed the absence of an effusion in the right hip and the presence of point tenderness over the right sacroiliac joint and suggested a Tc- 99 bone scan.

Meanwhile, results of the blood cultures were negative; throat and vaginal flora were normal with no growth of Neisseria gonorrhoeae.

On day 4 after admission the bone scan showed increased isotope uptake in the right sacroiliac area. After consultation with the rheumatologists treatment with acetylcalicylic acid was continued and indomethacin $25 \mathrm{mg}$ three times daily added to the regimen.

The patient's condition remained unchanged and on day 10 a Craig needle-biopsy of the right sacroiliac joint was performed. The material from the biopsy was submitted for routine aerobic and anaerobic culture, culture on Thayer-Martin medium for gonococci, culture for acid-fast bacilli, and histological studies. Suspicious colonies on ThayerMartin medium were evaluated by Gram staining and biochemical tests. 
On day 13, the patient continued to have pain; the treatment was changed from indomethacin to phenylbutazone $100 \mathrm{mg}$ three times daily. Further laboratory results showed a C3 complement level of $257 \mathrm{mg} / \mathrm{dl}$, a negative response for HLA-B27, and normal haemoglobin electrophoresis. The sacroiliac biopsy showed chronic, non-specific synovitis.

After four days' treatment with phenylbutazone, the patient's condition had improved slightly and she was able to bear weight on crutches despite pain in the right hip and sacroiliac region. On 13 October 1976 the patient was discharged; she was to continue taking phenylbutazone and acetylsalicylic acid.

On 15 October 1976, when she returned for followup examination, the biopsy culture showed growth of $N$ gonorrhoeae. The patient was readmitted and treatment with aqueous penicillin 500000 units i.v. every four hours was started and continued for 10 days. The patient's condition promptly improved. On day 10 after readmission, she was discharged free of symptoms and had a full range of movement in the right hip.

\section{Comment}

The unusual feature of this case is the involvement of the sacroiliac joint. Only eight reports relating to gonococcal arthritis since 1919 mention sacroiliac joint disease, confirming its rarity. $\mathrm{Michel}^{3}$ reported five cases of sacroiliac joint disease in his series of 100 patients and Wehrbein ${ }^{4}$ found 15 in a series of 610 cases. Later in the 1930s, Myers and Gwynn ${ }^{5}$ reported nine in their series of 85 cases, Keefer and Spink ${ }^{6}$ found eight cases in their study of 140 , and Burbacher and Weiland ${ }^{7}$ mentioned sacroiliac joint disease in their series of 58 cases. In 1949, Culp ${ }^{8}$ reported one case in his study of 200 cases, in 1942 Harkness 9 reported 17 out of 336 patients, and in 1974 Seifert ${ }^{10}$ reported severe sacroiliac joint pain in one of his 16 cases.

During the last decade, many of the classical concepts of gonococcal arthritis have changed, mostly because of a clearer understanding of the clinical features of this disease and a greater accuracy in diagnosis. ${ }^{11}$ Thus many of the earlier case reports may have contained a potential source of confusion with what we today recognise as Reiter's syndrome. Similarly, patients with Reiter's syndrome may have had $N$ gonorrhoeae coincidently identified in their genitourinary tract. This may account for the occasional case of gonococcal arthritis that failed to respond to penicillin therapy. ${ }^{212}$ Today we are able to separate Reiter's syndrome as a distinct clinical entity. Thus, even if Reiter's syndrome may have been a cause of confusion in these earlier reports, sacroiliac joint involvement was still an unusual occurrence.

In addition to joint involvement, this case indicates the importance of taking samples for culture from several sites to obtain a bacteriological diagnosis in a symptomatic patient. Although some authors suggest that treatment of gonococcal arthritis should be based on the clinical picture alone, it is also essential to make a bacteriological diagnosis, either by culture or by more invasive procedures such as joint aspiration or biopsy. This bacteriological information differentiates gonococcal infection from other types of articular disease.

\section{References}

1. Keiser H, Ruben FL, Wolinsky E, et al. Clinical terms of gonococcal arthritis. New Engl J Med 1968; 279: 234-40.

2. Wright V. Arthritis associated with venereal disease. A comparative study of gonococcal arthritis and Reiter's syndrome. Ann Rheum Dis 1963; 22: 77-90.

3. Michel LL. Infectious arthritis of urogenital focal origin. NY State J Med 1919; 109: 1124.

4. Wehrbein H. Gonococcus arthritis: a study of 610 cases. Surg Gynecol Obstet 1929; 49: 105-13.

5. Myers WK, Gwynn HB. Clinical features of gonococcal arthritis; observations in 85 cases. Med Ann DC 1935; 4: 194-97.

6. Keefer CS, Spink WW. Gonococcic arthritis: pathogenesis, mechanism of recovery and treatment. JAMA 1937; 109: 1448-53.

7. Burbacher CR, Weiland AH. Gonorrheal arthritis. J Fla Med Assoc 1938; 24: 433-6.

8. Culp OS. Treatment of gonococcal arthritis: analysis of 200 cases. J Urol 1940; 43: 737-65.

9. Harkness AH. Gonococcal arthritis. Med Press 1942; 207: $60-4$.

10. Seifert MH, Warin AP, Miller A. Articular and cutaneous manifestations of gonorrhoea. Review of sixteen cases. Ann Rheum Dis 1974; 33: 140-6.

11. Kushner I. Gonococcal arthritis. Med Times 1970; 98: 111-16.

12. Holmes KK, Counts GW, Geaty HN. Disseminated gonococcal infection. Ann Intern Med 1971; 74: 979-93. 